\title{
Laurence Aubry
}

\section{POUR UN ÉCLAIRAGE STYLISTIQUE DE L'CEUVRE DE FREUD : de l'opération médicale à la métaphore opérante dans la nouvelle édi- tion intégrale des Lettres à Wilhelm Fliess}

Cet article propose de reconsidérer les liens entre la psychanalyse, la littérature et les sciences du langage à partir d'une approche des textes analytiques centrée sur le détail de l'expression. Nous partons ici de la question de la métaphore, croisant celle de la traduction et de l'histoire des œuvres de Freud pour examiner un événement de lecture : la redécouverte des lettres à Flie $\beta$ dans la nouvelle traduction intégrale en français. Nous montrerons comment un complexe figural irradie à partir de la métaphore de l'opération, considérée comme une figure vive dans les énoncés concernant Emma Eckstein. Ainsi le style anticipe sur la découverte à venir de la cure, de l'Edipe et du transfert. Comme l'écrivain absolument moderne, l'écriture est en avant de la science qu'elle prévoit et annonce. Aussi la question du style renoue-t-elle un dialogue où psychanalyse et littérature trouvent à s'enrichir mutuellement dans le respect de leur altérité.

RELIEF 4 (1), 2010 - ISSN: 1873-5045. P85-101

http://www.revue-relief.org

URN:NBN:NL:UI:10-1-100952

Igitur, Utrecht Publishing \& Archiving Services

(C) The author keeps the copyright of this article

Depuis Freud, les psychanalystes usent de la métaphore pour parler de leur art. Ce qu'ils disent de la métaphore, aussi bien que son effet énonciatif dans leurs propres textes, continue selon nous d'enrichir aujourd'hui par ricochets l'approche de la littérature. La poursuite du dialogue fondateur, pour la psychanalyse, entre la nouvelle science de l'âme et les Lettres se manifeste par exemple dans l'attention prêtée actuellement au processus 
dynamique dans les études littéraires, cela tout particulièrement lorsqu'il y est question du style (Herschberg-Pierrot). Nous retrouverons ce mouvement dans le style de Freud à l'origine : tel qu'il se manifeste dans un texte préanalytique, récemment publié intégralement et traduit en français.

\section{Prolégomènes : une étude de style ou les rebonds d'une métaphore dans les lettres mentionnant Emma Eckstein}

On lit autrement La naissance de la psychanalyse avec le contrepoint des Lettres à Wilhelm Fließ. Mais le style de Freud dans cette correspondance est également transformé du fait de la restitution des lettres ou des fragments supprimés. La correspondance complète - où les lettres de Flie $\beta$, détruites, restent toujours en creux - fait sentir la force d'une censure opérant déjà au sein du psychisme de Freud. La publication tronquée initiale se réfracte en quelque sorte après-coup dans l'écriture de l'épistolier. Ainsi le refoulement se découvre dans son auto-analyse.

Il s'agira donc d'examiner, du fait même de l'histoire de cette correspondance, de sa publication et de ses exégèses, le sens que prend un entrelacs métaphorique dont la matrice "n'apparaîtra pas dans le texte " (Riffaterre 1982, 100). Plus exactement y insiste-elle par ce manque: telle une obstinée "manière de dire qui ne cesse de tourner autour d'un mot clé » réduit au statut de marque (Riffaterre 1983, 25). Dans les lettres 50 à 152 de la nouvelle édition, écrites entre le 29 août 1894 et le 29 décembre 1897, l'opération - référent ou base lexicale - se déplace "en réseau " (Ricœur, 1975, 307), par exemple dans le signifiant pus. Elle y forme des «complexes » (259) jouant aussi de la disposition des trois personnes : Freud (je), Flie $\beta(t u)$ et Eckstein (elle). En raison de son irradiation signifiante, nous montrerons qu'il s'agit d'une métaphore. Là encore, l'édition censurée redouble en le radicalisant ce travail du style, plus manifeste dans l'édition complète : effacée avec toutes les lettres ou les fragments mentionnant explicitement Emma Eckstein, la métaphore de l'opération est à la fois «filtre » qui fait voir et « masque qui déguise » (317).

Centrée sur le détail de l'expression, notre lecture stylistique a donc construit son objet à partir des morceaux de la correspondance caviardés 
par les premiers éditeurs. Elle renoue avec une "affaire» (Schur, 1975b) depuis interrogée par de nombreux commentateurs. Max Schur et Didier Anzieu l'ont rapportée à l'ambivalence de Freud envers Flie $\beta$. Le transfert de Freud apparaît massivement dans ses lettres, et l'on peut penser que l'auto-analyse a rétrospectivement participé à l'élaboration ultérieure du concept. En ce sens, le style de Freud gît dans la reconnaissance d'une métaphore latente effacée par les premiers éditeurs. L'écriture est en avance sur la découverte : absolument moderne.

Ainsi transfert et contre-transfert manquant à l'auto-analyse et ouvrant à l'CEdipe sont-ils indiqués dans l'écriture des Lettres à Wilhelm Fließ. Cela de façon exemplaire dans une métaphore associant au dynamisme de son processus celui du sens de son imageant. Le terme opération comporte en effet des sèmes présents dans les mots transfert et métaphore: "procès", "modification", "complexité". Au sens d'une opération chirurgicale - le même mot se retrouve alors en allemand et en français -, il peut référer dans ces lettres à des interventions du nez pratiquées par Flie $\beta$ aussi bien sur ses patients que lui-même et sur ses proches. Le mot opération revient donc comme le terme générique désignant un acte chirurgical effectué sur Freud (Freud 2006, 14711 140² ; Anzieu 1988, 54), sur Flie $\beta$ lui-même (Freud 2006, 162) ${ }^{3}$, enfin sur une patiente de Freud, Emma Eckstein (148-149) ${ }^{4}$. Trois opérations du nez, en l'espace de trois mois - entre février et avril 1895 -, dont les destins croisés essaiment dans les lettres à travers un réseau d'indices décroissant à mesure que « la correspondance des deux hommes prend de plus en plus l'allure d'un dialogue de sourds » (Anzieu 2000, 1211). Max Schur et Didier Anzieu s'accordent à reconnaître dans l'accident lié à l'opération d'Emma en 1895 l'élément déclencheur de l'émergence d'un aspect négatif dans le transfert amoureux de Freud sur Flie $\beta$. En décembre 1897, le conflit devient manifeste. Fort de la découverte de l'Edipe et de la sexualité infantile, Freud s'oppose alors aux thèses de Flie $\beta$, en particulier à la théorie qui lie la bisexualité à la bilatéralité en subordonnant sur un mode fantaisiste le psychique à l'organique. Flie $\beta$ date ainsi de janvier 1898 la rupture de leur amitié (Schur 1975a, 178). Sans se rejoindre exactement sur les différentes phases du déclin de ce transfert princeps, Max Schur et Didier Anzieu dévoilent les ambivalences et les aléas d'une 
liquidation restée incomplète. La rupture est cependant avérée après l'été 1900. Devenue convenue, leur correspondance cesse en juillet 1904.

La construction d'un corpus restreint nous a amené à sélectionner le complexe de l'opération qui court entre les trois protagonistes d'un drame œdipien déguisé. Il couvre donc la phase où le conflit trouve une forme. Il correspond à la première mention explicite d'Emma Eckstein dans la lettre du 4.3.1895, comme première patiente sur qui Flie $\beta$ tente « une opération qu'il n'a encore jamais pratiquée » (Freud 2006, 148 ; Anzieu 1988, 41 ; Masson, 95). La dernière se trouve dans la lettre du 12.12.1897 où elle figure comme ... thérapeute (Freud 2006, 364) : d'après Didier Anzieu, elle est « la première personne après Freud à avoir exercé la psychanalyse » $(1988,184)$. On la retrouverait parmi les trois exemples illustrant la négativité du transfert dans Analyse sans fin et analyse avec fin en 1937. Elle pourrait donc bien être, sinon «la "première patiente analysée" de Freud » (Masson, 75, 217), du moins « un des tout premiers cas de Freud traités par la méthode psychanalytique » (Anzieu 1988, 185).

Plutôt que celles qui mentionnent explicitement Emma Eckstein, nous avons choisi pour limites externes de notre corpus deux lettres marquées l'une par une métaphore, l'autre par une allusion en forme de mot-valise et de pirouette verbale: s'y consomme, puis s'y consume le transfert amoureux entre les deux hommes.

Dans le premier extrait, Freud réagit de façon très affective aux suites d'une opération que son ami a pratiquée sur sa propre personne :

Très cher ami,

Cela ne va vraiment plus du tout, allons-nous donc te voir disparaître tout entier dans le pus ? Encore et encore opérer, que diable, finis-en une fois pour toutes! (2006, $123)^{5}$

L'hyperbole qui fait peser sur Flie $\beta$ la menace d'un engloutissement putride se retrouve déplacée sur le versant intellectuel de la recherche et du lien. Elle est inversée dans son mécanisme et translatée sur Freud à l'autre pôle de la première partie privée de la lettre (la seconde étant dévolue à deux vignettes cliniques) : 
Mais à présent je dois supposer une fois de plus que tu seras assez en forme pour me débarrasser de la matière scientifique qui s'est accumulée. $(124)^{6}$

Au gré d'une répétition, d'une variation et d'un déplacement que la rhétorique nommerait une hypallage, des signes liés à l'opération ont fait émerger dans le texte un réseau métaphorique dont Emma formera dès son apparition le troisième nœud. Ainsi le modèle d'une métaphore matricielle pourra-t-il préfigurer par-delà les avatars d'un transfert homosexuel la découverte du triangle œdipien.

Quelques cent lettres plus loin, dans la condensation - métonymie, réduction et réduplication burlesque - où s'abrège et se déplace l'image du couple Flie $\beta$-Freud, est prédite la modalité brutale de leur rupture : «Bi-bi résonne à mes oreilles » $(369)^{7}$ signifie brutalement le Non! de Freud à son ami. Flie $\beta$ exige alors de lui le sacrifice de ses "découvertes » psychiques sur l'autel de ses propres hypothèses organicistes absurdes associant bisexualité et bilatéralité (Schur 1975a, 176).

Raccourcie à l'extrême, la formule Bi-bi est l'envers de la solution du conflit déployée dans les figures de la dernière lettre nommant Eckstein où se cèle ce qui, de cette relation à trois, demeurera inanalysé. Auparavant, les récurrences de la base pus (Eiter) apparaissaient corrélées aux projections énonciatives jouant de la disposition des marques des trois personnes. Derrière le pus, déterminé par la potentialité métaphorique de l'opération, pouvait «se lire en filigrane »-comme dans le rêve de l'injection faite à Irma mais transposée dans la langue d'une écriture - une « anticipation de la découverte à venir » (Anzieu 1988, 54).

Le réseau métaphorique ainsi mis à jour pointe des lieux du texte marqués d'ambivalence, où le transfert paraît en abyme dans des formes joignant le visible et l'invisible. Ainsi en est-il de cette opération déjà plusieurs fois évoquée, qui fait l'objet d'une dénégation dans la lettre censurée du 24.1.1895:

Nous ne sommes plus qu'à une semaine de l'opération ou du moins de ses préparatifs. Le temps a passé vite et j'évite volontiers de me demander de quel droit j'en attends autant. (Freud 2006, 140) ${ }^{8}$ 
Plus sensible encore en allemand qu'en français, la prétérition vise une intervention dont la référence demeure énigmatique, ensevelie semble-t-il à jamais dans le secret de son énonciation...

En 1966, Max Schur passe sous silence la lettre du 12.12.1897, qui mentionne Emma Ekstein comme analyste. Sa désignation n'y fait plus le jeu du couple Freud-Flie $\beta$, mais elle a apparaît comme sujet en position centrale. Selon une construction complexe, le signifiant paternel(le) (Vater) encadre un parallélisme entre Eckstein et sa patiente redoublé dans le jeu des pronoms :

\begin{abstract}
Ma confiance dans l'étiologie paternelle a beaucoup augmenté. Eckstein a directement traité sa patiente dans une intention critique, de façon à ne pas lui donner la moindre indication sur ce qui va sortir de l'inconscient, et elle a alors obtenu $\underline{\text { d'elle }}$ les mêmes scènes paternelles, etc. Accessoirement, la jeune fille va parfaitement bien. (Freud 2006, 364) ${ }^{9}$
\end{abstract}

Avec le narcissisme, n'est-ce pas l'importance pour la psychanalyse du lien homosexuel qu'annoncent ces lettres de Freud à Flie $\beta$ ? Nous soutenons que leur force pour le psychisme tient également au style dans lequel ces notions sont pré-vues.

Cette étude ouvre quelques pistes pour un questionnement croisé de la théorie de la métaphore, de la psychanalyse à travers le style de ses écrits, et de la question de sa traduction. Nous les indiquerons plus succinctement :

\title{
1. Métaphore et traduction :éclairages réciproques de la psychanalyse et de l'herméneutique
}

« Le style ici est créateur de l'objet, c'est-à-dire que contenant et contenu ne sont plus séparables, sont même interchangeables » (Roustang, 33). Dans la métaphore, leur léger décalage inscrit au creux du signe-figure la même temporalité paradoxale et énigmatique qu'évoque en psychanalyse le phénomène de l'après-coup, où s'épanche une fiction. Parmi ces images dont «l'irruption [...] au sein même des concepts freudiens les plus abstraits confère $[\ldots]$ au discours psychanalytique une hétérogénéité provocante, 
une véracité compromettante et révolutionnaire (Altounian, 90), nous avons justement sélectionné la moins visible... Nous suivons en cela Paul Ricœur pour qui, dans la vraie métaphore, l'analogie se dérobe sans que la figure ne s'abolisse dans le lexique. Sa forme vive se déploie en réseau et crée un complexe discret, voire secret : ses lacs, comme son scandale, ne se révèlent qu'a posteriori, au décours d'une lecture peu ou prou qualifiable de stylistique - que celle-ci soit d'un amateur ou bien d'un savant, selon le subtil dosage qui fait un interprète. Les figures de l'analogie - comparaison, métaphore in praesentia, métaphore filée, allégorie, etc. - relèvent davantage d'une analyse rhétorique en terme d'effets; tandis que la métaphore encore en germe dans le discours saura indiquer ce « rapport étroit entre l'aptitude à l'ambiguïté dans l'écoute analytique et celle que met en acte toute écriture » (Altounian, 112). Elle soulève pour le traducteur une autre difficulté que la restitution de la «densité » de « la morphologie ou la syntaxe », ou d'une " originalité » propre à «l'agencement interne des signifiants » (Altounian, 89-90).

\section{Perspectives de la métaphore dans l'écriture de la psychanalyse: les royaumes intermédiaires et le narcissisme du langage}

Pour rendre compte de la métaphore au nœud de l'articulation entre langue et psychanalyse, nous proposons de la réentendre telle que, réciproquement, certains psychanalystes l'écoutent avec la linguistique poststructurale, et telle que certains spécialistes du texte la lisent avec les acquis et les pistes ouvertes par la psychanalyse. Cela implique de considérer, avec Umberto Eco, que la métaphore est d'abord une "interprétation » (152-153), avec Roland Barthes, qu'elle est du côté de l'écrit comme la «littérature » (16-21), avec Paul Ricœur, qu'elle est un moment, entre sensation et compréhension, où se déguste "l'incertitude de la trace » (Gantheret, 209). Elle est "une ébauche du temps » (212), dans cette langue où «les mots rêvent » (Bachelard, 16) dont Roman Jakobson a rendu compte du point de vue linguistique.

A travers la métaphore, se signifient quelques dimensions essentielles du psychisme humain, réfractées dans le signe verbal, et interrogées par la psychanalyse. Notre réflexion sur le style de Freud à l'aube de sa pensée 
nous a conduite à isoler parmi elles, dans le cadre de cette étude, des termes signifiant l'entre-deux, le conflit, et le mouvement.

Dans la métaphore, il nous semble en effet que la psychanalyse et la linguistique peuvent indiquer l'intermédiaire. Là se signe le lien entre le langage et le narcissisme - « cette organisation [...] instable, frontalière » entre « autoérotisme » et "phase œdipienne ». Il recoupe l'intérêt de Freud pour « la signifiance»: selon Julia Kristeva, celle-ci n'aurait « rien de linguistique », mais renverrait à l'articulation « entre acte et représentation, entre irreprésentable et contrat symbolique autour de l'autorité » (72-73). « Ni langage ni pulsion, mais qui [les] surdétermine ", la signifiance comme la vraie métaphore veulent indiquer ce qui ouvre dans le retour de la trace sensible devenant un signe, «comme une narration » (94). L'entre-deux c'est aussi - et particulièrement pour Freud à l'époque de la correspondance avec Flie $\beta$ ce " phénomène psychique qui soit intermédiaire » entre le normal et le pathologique: "c'est le rêve» qui, selon la belle formule de Didier Anzieu, « est une hallucination brève » $(1988,32)$. C'est le « tourment » (Freud 2006, 167) ${ }^{10}$ dont témoigne le Projet d'une psychologie, qui exprime la tension entre intuition et science, thérapie et théorie, physiologie et psychisme : c'est le signe du conflit affectif individuel hérité de l'infantile, dont Freud découvre le paradigme en même temps que la lecture des Lettres à Flie $\beta$ nous le dévoile "luttant contre certains effets dynamiques de sa propre analyse » (dans Freud 1956, 28). C'est aussi un néologisme, comprenant la préposition allemande zwischen, dont les divergences et les hésitations des différents commentateurs soulignent l'ambivalence. Ces débats indiquent aussi l'importance de cette métaphore du « royaume intermédiaire » (Freud 2006, $234)^{11}$, que Didier Anzieu préfère traduire «royaume de l'entre-deux» $(1988$, 81). Sous la plume de Paul Ricœur, on la retrouve à plus d'un titre pour caractériser la métaphore dans le discours, articulée aux notions de tension et de dynamisme...

Du côté de la psychanalyse, l'intermédiaire et la tension évoquent, sur le modèle du rêve, la complexité de l'articulation entre la pratique et la théorie, la cure et son compte-rendu, la clinique et la métapsychologie, le début et la fin d'une analyse. Ainsi la question du temps et de la vérité en psychanalyse peut-elle se cristalliser dans « le problème d'une écriture de la clinique analytique, en droit impossible [...] parce que nous n'y sommes 
plus » (Gantheret, 160). Elle se reconnaît encore dans le travail de la métaphore, en tant qu'il représente la déformation du souvenir. Selon nous, c'est donc par une approche stylistique empruntant le détour de l'écriture que le langage littéraire et la psychanalyse pourront le mieux continuer de s'éclairer mutuellement.

\section{La métaphore ou la pré-diction du style: un sens par excès ou un signe au-delà}

En exergue à cette question posée au style de Freud, notons un paradoxe qui a cours, nous semble-t-il, aussi bien chez les psychanalystes que chez les linguistes ou les littéraires. On redit l'importance de l'écriture au regard de la cure (Gantheret, Pontalis), on reconnaît la valeur heuristique et réflexive de la langue de Freud relativement à son objet; mais on note, comme à regret: «la psychanalyse dans son rapport au style ne vient que rarement sur l'avant-scène » (Roustang, 9). Discriminant le pratique du théorique, le style de l'analyste écrivain peut cependant éclairer ce qu'a aussi inventé Freud: "le style de l'analyse qui, dans la cure, produit de l'analyse » (Roustang, 46). En ce sens, il peut être un mode d'accès privilégié à la clinique. S'il est rarement ou superficiellement interrogé, c'est qu'il manifeste un conflit. Ainsi rejoint-il la métaphore, qui emblématise la tension dans son processus même. Relevant d'une défense au sens psychanalytique - homologue en cela à ce que Freud signale à plusieurs reprises dans ses lettres à Flie $\beta$ comme le problème dont la résolution le taraude -, le style néanmoins insiste dans les formations de l'inconscient. En cela, il croise le sort qui lui est fait dans les débats actuels tant en linguistique qu'en littérature (Molinié, Cahné) ${ }^{12}$.

«Impossible d'échapper aux métaphores du texte » nous dit Janine Altounian (28). Aussi sont-elles la figure dans laquelle convergent la psychanalyse, l'écriture de Freud et le langage créateur. À contre courant du postulat d'un «clivage entre l'expérience de la psychanalyse et la lecture de ses textes ", répétant "l'écart existant entre la version originale d'un écrit de Freud psychanalyste et celle de sa traduction en langue étrangère » (Altounian, 84), comme aussi la différence entre le thérapeutique et le scientifique (Arrivé, 15-16), nous saisissons la réflexivité de la langue de Freud à un au- 
tre niveau. Nommons le stylistique. Il nous semble en effet que si la distinction des aspects d'une pratique est indispensable au traducteur, au linguiste et sans doute parfois à l'analyste, le lecteur, lui, est transformé par la rencontre avec le texte de Freud pourvu qu'elle soir opérante, à l'instar de l'analysant vivant et mesurant les changements dans sa cure. Ainsi l'écriture de Freud n'indique pas seulement « une pensée qui réfléchit sur ses propres outils» (Altounian, 71). Et si se retrouvent, dans les «mots qui, tout en étant ceux de la théorie [...] ressemblent à ceux de l'acte thérapeutique », quelque chose de l'acte poétique, c'est par ce que leur réflexion indiquent en excès ou au-delà de la signification.

\section{Différence de la métaphore, dynamisme de la langue, dynamisme dans la psyché : au miroir d'une figure de style}

Pour étudier ce procès inscrit dans la langue au niveau du style, nous avons donc choisi de privilégier ce que Paul Ricœur nomme la métaphore vive. Dans l'écriture de Freud comme ailleurs, elle ne saurait se confondre avec ce que ses principaux commentateurs regroupent souvent sous le terme de métaphore, et qui englobe en réalité les autres figures de l'analogie: comparaison, allégorie en particulier. Walter Muschg comme Janine Altounian privilégient eux les effets poétiques d'une «langue imagée » (Muschg, 90), seule propre à rendre la « plasticité » (Altounian, 36) de la vie psychique. Aussi optent-ils pour une perspective rhétorique soulignant, avec sa valeur mimétique, l'effet $\mathrm{d}^{\prime}$ « un style dérogeant aux convenances » (Altounian, 90). S'y manifeste l'ethos d'un Freud tantôt ou à la fois écrivain, scientifique, psychologue, thérapeute, enseignant, découvreur... Or, la comparaison, l'analogie, la métaphore filée lorsqu'elle confine au parallèle, voire l'appropriation d'un trope d'usage, sont chez Freud plutôt statiques, descriptives. Leurs motivations sont à dominante explicative et didactique, leurs fonctions manifestement figuratives. "La comparaison méthodique » ou " rapide», «la métaphore arrondie et devenue autonome » sont parmi les «caractéristiques les plus personnelles de l'écrivain Freud » (Muschg, 90).

Mais c'est toutefois « comme variations d'un motif originaire » $(93)^{13}$ qu'elles participent d'un trait de son style emblématisé dans la vraie méta- 
phore: la dimension dynamique. Cette perspective est importante dans la pensée analytique, où elle coexiste avec les points de vue économique et topique. Cette qualité est aussi une caractéristique de la langue allemande souvent mentionnée. La vraie métaphore se marque ainsi dans l'écriture à la fois comme figure de réflexion et signe de science fiction à valeur d'anticipation, décelé(e) après-coup dans le temps de sa lecture. Freud luimême indiquait-il autre chose lorsqu'il soulignait que l'écrivain «a été de tout temps le précurseur de la science et par là aussi celui de la psychologie scientifique » $(1986 \mathrm{a}, 184)$ ?

Freud reconnaît progressivement l'importance « du point de vue dynamique en psychologie » (Anzieu 1988, 28) et accorde de plus en plus de poids au conflit psychique dont il conçoit la genèse. Dans le même temps, il privilégie dans son écriture, parmi les tropes, celui le plus chargé d'une valeur dynamique, et parmi les métaphores ou les figures elles-mêmes, celles où s'actualise ce caractère associant le mouvement, la transformation et la réflexion. Selon Barthes, c'est là le propre $d^{\prime}$ « un discours qui n'est plus épistémologique, mais dramatique » (19). Walter Muschg relève ainsi la fréquence des métaphores verbales signifiant une action transitive - « façonner, corroborer, faire voler en éclats, ériger et rétablir, enregistrer »- ou encore des noms métaphoriques, souvent déverbaux, qui impliquent un procès : le fameux «déclin» (89-90). Nommons aussi les " "barrages" : pudeur, dégoût et morale, qui "contiennent" la pulsion sexuelle de l'adulte ».

Commentant les emplois du préfixe nach, de Nachträglich (après-coup), Jacques Schotte remarque «qu'en allemand la résonance verbale, processive, et donc une référence constante à cette dimension temporelle, sont beaucoup plus qu'en français l'apanage également de substantifs » (116). Aussi la métaphore fournit-elle " un exemple typique de l'usage significatif par Freud, de la "tendance phénoméniste et dynamique" inscrite au cœur de la structure de l'allemand ». Elle résonne alors avec les principes fondamentaux de la psychanalyse. Elle converge avec tous les termes ou traits de style qui « renvoient ainsi de multiples façons et avec des nuances très fines à un problème fondamental unique : celui des divers modes de temporalisation de l'histoire personnelle" ». Ainsi de la narrativisation de la métaphore, « que Freud développe en anecdotes ou en nouvelles » (Muschg, 93), ou de la répétition-variation jouant sur une intertextualité interne où 
l'œuvre dessine sa cohérence. La célèbre métaphore du chemin (Weg) est emblématique de son processus: pour Walter Muschg, elle «transforme [...] le sentiment du "chemin" en une construction d'une transparence cristalline » (106) ; pour Jacques Schotte, elle reflète « le procès analytique luimême » (63), le cheminement de la cure. Elle traduit enfin, selon Janine Altounian, la «dynamique guidée par l'inconscient» (31): «la dynamique des conditions de création de la démarche analytique mais aussi celle-là même de la cure instituée par elle, la dynamique d'une genèse et de sa transmission ».

Les métaphores innervent (95-96) ${ }^{14}$ réflexivement l'écriture de Freud pour indiquer au-delà, ce qui dans le langage nous échappe. Cela a à voir avec le corps, l'affect, la sexualité. Cela rejoint la parole en sa genèse et dans son décalage : l'inconscient $n^{\prime} e s t$ pas le langage. Les métaphores pointent la nécessité $d^{\prime}$ '« une certaine prise en compte de l'irreprésentable, de l'inverbalisable opérant au sein de l'appareil psychique et se marquant ainsi brutalement dans le contre-transfert » (Kristeva, 97-98). C'est dans un registre proche, nous semble-t-il, que Georges Molinié tente de rendre compte du caractère sexuel de l'expérience de lecture dans ce qu'il appelle le régime d'art ou la réception littéraire (181-230).

\section{La psychanalyse et la métaphore: d'une lecture conservatrice à une fi- gure du transfert}

Freud ne parle jamais de métaphore, non plus qu'il n'emprunte, dans l'ensemble de son œuvre, au lexique de la tropologie (Dürrenmatt 78). La lettre du 15.10.1897 (2006, 343-344), par exemple, est consacrée à un souvenir et à une découverte centrale dans son auto-analyse : rien moins que la valeur paradigmatique pour le psychisme humain d'CEdipe Roi. Ce que Freud décrit en interprétant la base kast dans Kasten ("coffre ») et eingekastelt («coffrer») n'est pas la polysémie ni "l'indétermination» (Ricœur, $1975,25)$ propre à la vraie métaphore, mais le double sens où chacune des deux acceptions du radical convoquées - le propre et le figuré - conserve son autonomie et son intégrité. Son interprétation indique clairement le lien, mais aussi la séparation, entre deux domaines distincts. Elle relève donc d'une lecture alignée sur la comparaison et d'un «fonctionnement essen- 
tiellement analogique » (Dürrenmatt, 16). À la suite de Paul Ricœur relisant d'Aristote, les perspectives contemporaines s'accordent à en distinguer le processus métaphorique.

Quelques lettres plus loin, alors qu'il évoque "l'indétermination verbale » utilisée pour « la représentation de contrainte », Freud s'émerveille de reconnaître un procédé d'invention lexicale qui n'est pas la métaphore, mais la catachrèse : "C'est tout à fait fou, mais tout à fait analogue au processus d'après lequel des mots prennent une signification figurée dès que se présentent des concepts nouveaux ayant besoin d'être désignés » (2006, 366-367 $)^{15}$. Sa conception du langage épouse donc les différentes théories qui, selon Paul Ricœur, ont pu égarer dans la compréhension de la métaphore ou du moins en masquer une dimension essentielle. En linguistique comme en philosophie, elles font aujourd'hui l'objet d'un débat (Dürrenmatt, 15-21, 69-76).

En 1996, F. Gantheret peut dire de ces mots débusqués dans le symptôme, le lapsus, le rêve, le geste, le corps ou la mémoire de l'analyste « qui, sans le savoir, a perçu la part de mystère qui s'attachait à une image, à une expression pourtant fort banales » (109): «je ne pense pas que l'analogie constitue à elle seule le matériau de l'interprétation». Il y faut un temps de plus : recherche d'une «qualité » pour cela «qui manquera à la trace» (211). Ce manque pourra s'inscrire par exemple dans un rythme, "quête pulsatile du mot [...] par laquelle le langage se centre... sur lui-même ».

Depuis Freud, certains psychanalystes ont aussi rapproché métaphore et transfert. Celui-ci vient ainsi selon Jacques Schotte " articuler pour tous l'allure métaphorique de l'existence humaine» (64). En tant qu'analysante, Janine Altounian le rapproche à demi-mot de l'activité du traducteur (85). La caution venue de la langue est le radical über en allemand - le préfixe trans signifiant le " passage au-delà d'une frontière » ou le «changement» dans les langues latines - communs à Übersetzung et Übertragen (soit « traduction » et « transfert»).

Ajoutons qu'il est courant de décrire la métaphore en terme de transfert: au sens d'un transport de significations entre domaines sémantiques, entre le connu et l'inconnu, entre la langue et l'énoncé. Le «dynamisme de la signification elle-même » (Ricœur, 1975, 379) paraît alors redoubler le désir du sujet inscrit dans le mot : inventer dans le langage. 
Enfin, pour éclairer cette fois le paradoxe de l'écriture psychanalytique, Jean-Bertrand Pontalis assigne au texte la charge «d'instituer avec [son] lecteur quelque chose comme un transfert, c'est-à-dire un lien au présent avec un absent» (109). En ce sens, elle est pour le psychanalyste qui écrit " une mise à l'épreuve " (102) de sa pratique et un autre lieu où se transportent les traces de son propre transfert. Contre projection, l'écriture permettrait-elle l'inscription de ces «prises» (Pontalis, 82) hétéroclites « dans la chair des mots » (Gantheret, 165) ?

\section{Conclusion : le style de Freud ou une modernité au secret}

Ainsi, par la lecture de quelques lettres centrée sur une métaphore ne se laissant pas d'emblée deviner comme telle, avons-nous voulu montrer comment se manifeste à très bas bruit le cheminement de Freud dans ses écrits. Entre énergie et signifiance, ce trajet à double sens réalise selon Paul Ricœur une herméneutique dynamique propre à la psychanalyse (1965, 72-79). Anecdote exhaussée par la censure des lettres où elle somnole, la métaphore de l'opération prépare le mouvement de bascule qu'elle signifie obliquement. Du geste médical dans la référence, elle opère le passage au signe réflexif d'une écriture et d'une technique psychanalytique opérantes dans la métaphore. Elle transporte enfin l'expression du lien conflictuel à Flie $\beta$ : $\mathrm{s}^{\prime} \mathrm{y}$ annoncent la force et la valeur dynamique de la relation transféro-contre transférentielle dans la cure.

Pour autant qu'elle soit vive, partant poétique, la métaphore dans l'écriture de Freud est ainsi une force qui indique la part du littéraire dans l'œuvre, à l'œuvre dans le style. Elle est ainsi un signe privilégié pour dire la tension au cœur de la psychanalyse et de sa métapsychologie: en tant qu'elles sont nées de l'abandon de la neurotica, là où se termine aussi la relation épistolaire entre Freud et Flie $\beta$.

\section{Notes}

1. Lettre du 4.3.1895. Nous remercions chaleureusement Mme Sylvie le Moël, maître de conférences en langue et littérature allemande, pour avoir vérifié avec nous la pertinence de nos analyses à partir de la traduction, systématiquement confrontées à l'original (Freud 1986b). 
2. Lettre du 24.1.95.

3. Lettre du 20.4.1895.

4. Lettre du 4.3.1895.

5. Lettre du 29.8.1894: «Liebster Freund! Das ist doch nicht mehr schön, gehst Du uns denn ganz im Eiter auf? Wieder und wieder operieren, zum Teufel, nun werd' einmal fertig » (Freud, 1986b, 89). Les segments de l'énoncé participant du complexe métaphorique de l'opération sont indiqués en italiques. Les termes qui en portent particulièrement la signifiance sont en sus surlignés en français comme en allemand : très fort, le verbe aufgehen peut signifier « crever » en parlant d'un abcès. Didier Anzieu rappelle que les deux amis «souffrent tous deux du nez » et «parlent avec abondance et complaisance de cet organe qu'ils ont malade en commun [:] Ils sont en quelque sorte liés par le nez » (1988, 55). Ils partagent en réalité un complexe de symptômes, largement commentés dans les lettres, où Flie $\beta$ reconnaît « des équivalents menstruels » (Anzieu 2000, 1207).

6. " Jetzt muß ich aber wieder annehmen, Du seist ganz fesch, und mich der angehäuften Wissenschaftlichkeit entledigen » (1986b, 90). C'est le verbe angehäuften (« accumuler») qui entre en écho avec la métaphore du début de la lettre. Sans être métaphorique, entledigen ("débarrasser ») s'inscrit dans le même réseau marquant un mouvement de concrétisation, tandis que Wissenchaftlichkeit - à la lettre « scientificité », « fondements scientifiques» - ne connote pas la «matière » de la traduction française : la métaphore se répond certes dans les deux langues, mais au gré d'un déplacement entre les signes. Une étude de la base de sens concret häuften («tas », «pile»), dont l'emploi métaphorique est particulièrement fréquent dans ces lettres, permettrait de compléter notre analyse.

7. Lettre du 29.12.1897 : «Bi-Bi tönt mir in den Ohren » (1986b, 316).

8. " Es trennt uns nur noch eine Woche von der Operation oder wenigstens von ihren Vorarbeiten. Die Zeit ist schnell vergangen, und ich weiche gern einer Selbstprüfung aus, mit welchem Recht ich soviel von ihr erwarte. » (1986b, 105-106). Les termes allemands sont plus forts, surtout sous la plume de Freud au moment de son auto-analyse systématique: ausweichen signifie " éviter de », " esquiver », et selbstprüfen "s'examiner soi-même ».

9. "Mein Vertrauen in die Vaterätiologie ist sehr gestiegen. Die Eckstein hat ihre Patientin direkt in kritischer Absicht so behandelt, da $\beta$ sie $\underline{i h r}$ nicht die leiseste Andeutung gegeben, was aus dem Unbewußten kommen wird, und von $\underline{i h r}$ dabei die identischen Vaterszenen u. dgl. erhalten. Nebenbei geht es dem jungen Mädchen vortrefflich » (1986b, 312). Cette fois c'est entre les signifiants au sens saussurien que le jeu de la répétition des pronoms, pour le son et pour la place, a permuté entre l'allemand et le français.

10. Lettre du 25.5.95.

11. Lettre du 16.4.96.

12. Voir aussi l'intervention de Christelle Reggiani : «Les stratégies du style. Usages du mot dans le discours critique contemporain ", Actes du colloque Style, langue et société, dir. Éric Bordas, Georges Molinié, Centre Culturel, International de Cerisy-la-Salle, 10-17 septembre 2009, à paraître. 
13. Le critique cautionne son propos par une citation de Freud extraite de La question de l'analyse profane: "Mais nous devons aussi perpétuellement changer de comparaisons, car aucune ne nous soutient assez longtemps » (cité dans Muschg, 90-91).

14.L'auteur signale l'emprunt de cette métaphore de l'innervation à L'Interprétation $d u$ rêve, pour désigner ce qui dans le style de Freud traduit le «mode de représentation dynamique ».

15. Lettre du 22.12.1997.

\section{Ouvrages cités}

Didier Anzieu, L'auto-analyse de Freud et la découverte de la psychanalyse, Paris, Presses Universitaires de France, 1988 (3e éd. refondue, 1e éd. 1959).

Didiern Anzieu, «Vues nouvelles sur l'auto-analyse de Freud et la découverte de la psychanalyse », Revue Française de psychanalyse, LXIV, 4, 2000, 1201-1216.

Michel Arrivé, Langage et psychanalyse, linguistique et inconscient, Paris, Presses Universitaires de France, 1994.

Janine Altounian, L'écriture de Freud. Traversée traumatique et traduction, Paris, Presses Universitaires de France, 2003.

Roland Bachelard, Poétique de la rêverie, Paris, Presses Universitaires de France, 1960.

Roland Barthes, Leçon, Paris, Éditions du Seuil (coll. « Points essais »), 1978.

Umberto Eco, «Sur l'interprétation des métaphores », dans Les Limites de l'interprétation, Paris, Bernard Grasset (coll. « Le Livre de Poche »), 1992 (éd. orig.1990), 152-174.

Sigmund Freud (S), La naissance de la psychanalyse, Paris, Presses Universitaires de France, 1956 (éd. orig. 1950).

Sigmund Freud, « Analyse avec fin et analyse sans fin », dans Résultats, Idées, Problèmes II 1921-1938, Paris, Presses Universitaires de France, 1985 (éd. orig. 1937), 231-268.

Sigmund Freud, Le délire et les rêves dans la Gradiva de W. Jensen, Paris, Gallimard (coll. « Folio essais »), 1986 (éd. orig. 1907).

Sigmund Freud, Briefe an Wilhelm Fließ 1887-1904, Ungekürzte Ausgabe, Frankfurt am Main, S. Fischer Verlag, 1986 (1 ère éd en anglais 1985).

Sigmund Freud, L'interprétation du rêve. CEuvres complètes. Psychanalyse, t. IV, Paris, Presses Universitaires de France, 2003 (éd. orig. 1899 [1900]).

Sigmund Freud, Lettres à Wilhelm Fließ 1887 1904, Édition complète, Paris, Presses Universitaires de France, 2006 (1 ère éd. en angl. 1985).

François Gantheret, Moi, Monde, Mots, Paris, Éditions Gallimard, 1996.

Anne Herschberg Pierrot, Le style en mouvement. Littérature et art, Paris, Éditions Belin, 2005. Julia Kristeva, "Les métamorphoses du "langage" dans la découverte freudienne (Les modèles freudiens du langage) ", dans Sens et non-sens de la révolte. Pouvoirs et limites de la psychanalyse, t. I, Paris, Librairie Arthème Fayard (coll. « Le Livre de poche), 1996, 51-102. 
Jean Laplanche, Jean-Bertrand Pontalis, Vocabulaire de la psychanalyse, Paris, Presses Universitaires de France (coll. «Quadrige »), 2002 (1 ère éd. 1967).

Jeffrey Moussaieff Masson, «Freud, Flie $\beta$ et Emma Eckstein » et « Appendice. Freud et Emma Eckstein », dans Le réel escamoté. Le renoncement de Freud à la théorie de la séduction, Paris, Éditions Aubier Montaigne, 1984, 73-122 et 205-221.

Georges Molinié, Pierre Cahné (dir.), Qu'est-ce que le style ?, Paris, Presses Universitaires de France, 1994.

Georges Molinié, «Le simulacre - Le corps », dans Sémiostylistique. L'effet de l'art, Paris, Presses Universitaires de France, 1998, 181-230.

Walter Muschg, «Freud écrivain », La Psychanalyse, 5, 1959, 69-124 (éd. orig. 1930).

Jean-Bertrand Pontalis, La force d'attraction, Paris, Éditions du Seuil, 1990.

Paul Ricœur, De l'interprétation. Essai sur Freud, Paris, Éditions du Seuil, 1965.

Paul Ricœur, La métaphore vive, Paris, Éditions du Seuil, 1975.

Michel Riffaterre, «L'illusion référentielle », dans G. Genette , T. Todorov (dir.), Littérature et réalité, Paris, Éditions du Seuil (coll. « Points essais »), 1982, 91-118 (éd. orig. 1978).

Michel Riffaterre, Sémiotique de la poésie, Paris, Éditions du Seuil, 1983 (éd. orig. 1978).

François Roustang, "Du style de Freud », dans ... Elle ne le lâche plus, Paris, Éditions de Minuit, 1980, 9-46.

Jacques Schotte, «Introduction à la lecture de “Freud écrivain” », La Psychanalyse, 5, 1959, 5168.

Max Schur, La mort dans la vie de Freud, Paris, Gallimard, 1975 (éd. orig. 1972).

Max Schur, "L'affaire Emma. Lettres inédites de Freud à Flie $\beta$, en complément aux restes diurnes du rêve de l'injection faite à Irma », Etudes Freudiennes, 15-16, Figures de Freud, 1975, 151-182 (éd. orig. 1966).

\section{Notice bio/bibliographique}

Laurence Aubry est maître de conférences spécialisée en stylistique à l'Université de Perpignan, rattachée à l'équipe d'accueil Sens, textes, histoire dirigée par Olivier Soutet à l'Université Paris Sorbonne - Paris IV. Après une thèse sur la poétique de Jules Supervielle (à paraître aux Éditions Honoré Champion), elle a consacré plusieurs articles à une approche originale de la question du style croisant littérature et psychanalyse. 